Journal of Ancient Philosophy Vol. I 2010 Issue 1

\title{
La predicazione delle differenze nelle Categorie di Aristotele
}

Barbara Botter (PUC-Rio)

\begin{abstract}
In chapter 3 of the Categories Aristotle introduces a principle that is widely taken to assert the transitivity of the said-of relation. In this paper we shall show than something different may be going on in the transitivity principle that straightforward invocation of the transitivity of the said-of relation.
\end{abstract}

Key words: transitivity relation, difference (diaphorai), predication.

\section{La relazione di transitività}

In questo articolo ci proponiamo di discutere il significato del principio della transitività della predicazione nel contesto specifico delle Categorie e le conseguenze che esso comporta nella predicazione delle differenze (diaphorai).

Nel capitolo 3 delle Categorie Aristotele enuncia i requisiti che caratterizzano il principio della transitività della predicazione:

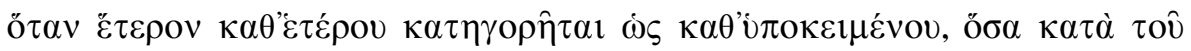

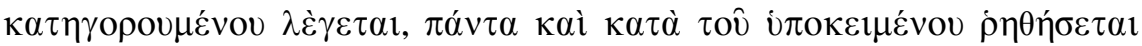

Quando una cosa si predica di un'altra come di un sostrato ${ }^{l}$ (hos kath'hupokeimenou), tutto ciò che si afferma del predicato, dovrà essere affermato anche del sostrato.

Secondo l'interpretazione tradizionale, ciò che questo principio si propone di mostrare è che la relazione tra la sostanza prima e la specie è della stessa natura che la relazione tra la specie e il genere: Aristotele non distingue, perciò, la relazione tra il sostrato (hupokeimenon) e il suo genere, e la relazione di una specie al suo genere, ossia, non distingue tra appartenenza e inclusione.

\footnotetext{
${ }^{1}$ Sostrato: con questo termine vogliamo indicare la sovrapposizione tra significato logico del soggetto di una proposizione e significato ontologico dell'ente capace di sussistere in sé.
} 
Quando Aristotele espone la regola della transitività della predicazione non usa il vocabolario dei generi e delle specie, ma esso si può facilmente dedurre da un passo degli Analitici Primi ${ }^{2}$ : ciò che si predica del genere si predica anche della specie, ciò che si predica della specie si predica anche delle differenze ma non necessariamente del genere, ciò che si predica delle differenze si predica anche degli individui, ma non necessariamente delle specie superiori e del genere. In altri termini, ogni egiziano o greco sarà uomo, ed ogni uomo sarà animale, ma non ogni animale sarà uomo né ogni animale sarà egiziano o greco. È per questo che Aristotele afferma: "Le sostanze prime accolgono la definizione delle specie e dei generi, e la specie accoglie la definizione del genere

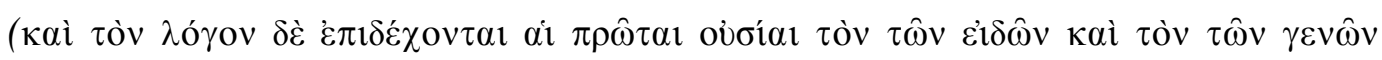

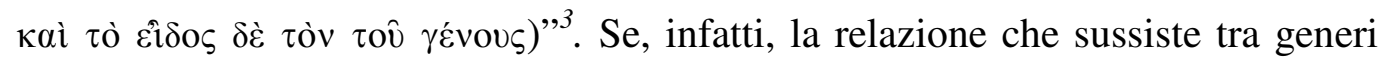
(G) specie (S) e sostanze prime (SP) è quella rispettivamente di inclusione e appartenenza, vale naturalmente la legge transitiva per cui se SP è incluso in $S$ e $S$ appartiene a $\mathrm{G}$ allora SP è incluso in G. Va da sé che, come ogni relazione di inclusione e appartenenza, la relazione non è reversibile: va preservata la direzione dell'operazione. È per questa ragione che se ogni greco è uomo ed ogni uomo è animale, non ogni animale è uomo e non ogni animale è greco ${ }^{4}$.

A partire dal capitolo 3 fino al capitolo 5 incluso, si percepisce uno iato, un décalage, che è costitutivo dell'operazione di predicare: l'operazione di predicare è asimmetrica, per così dire. Non sono dei termini posti sullo stesso piano che l'operazione del predicare lega. Si tratta sempre di un termine inferiore all'altro dal punto di vista del carattere concreto, e superiore all'altro dal punto di vista dell'universale ${ }^{5}$, come ha perfettamente colto Ammonio ${ }^{6}$.

La legge della transitività della predicazione fonda la struttura stessa che sta alla base dell'articolarsi dei generi in specie e sottospecie e che deriva dal

\footnotetext{
2 Arist., $A \operatorname{Pr}$ I 27, 43a25 sg.

3 Arist., Cat. 3b2-3.

${ }^{4}$ Questa tesi è esplicitamente enunciata al capitolo 5 delle Categorie (3b2-7), sulla base della formula impiegata a 1b10-12. Tradizionalmente si legge 5, 3b5-8 come una applicazione del principio della transitività della predicazione.

${ }^{5}$ Cfr. Porfírio, Isagoge II 14.

${ }^{6}$ Ammonio ha perfettamente colto questo tratto. Egli dichiara che l'obiettivo di Aristotele era di fornire un insegnamento "sui generi e le specie e di mostrare che le specie sono il soggetto dei generi, mentre i generi sono predicati delle specie". Ammonio continua come segue: "C'est pourqoui les individus sont seulement sujets, les intermédiaires [espèces et genres] sujets pour ceux qui les précèdent et prédiqué de ceux qui les suivent, et les suprêmes seulement prédiqués. Donc, comme lesdits vocables (phônai) désignent les genres suprêmes, en ce qu'ils sont toujours prédiqués et jamais sujets, il a intitulé son <livre> Les Catégories », Ammo nio, In Cat. p. 13, 13-19, citato dalla traduzione francese di de Libera 1998, p. XXVI, nota 33.
} 
perfezionamento della diairesi platonica. Del resto, il genere esprime un carattere comune alle specie sottostanti, le specie un carattere comune alle sottospecie e queste ultime un carattere comune agli individui. Così come è vero anche il contrario, ossia, l'individuo è parte di una sottospecie, ed essa di una specie, ed essa di un universale e questo ultimo è parte di un universale più ampio fino all'universale massimo, ossia quello che funge solo da predicato e mai da soggetto.

Patzig (Patzig 1968), Ackrill e Oehler, nei loro rispettivi commenti alle Categorie, ritengono che le argomentazioni che ci occupano in questo passo delle Categorie lascino intravvedere alcune strutture che in seguito appariranno negli Analitici Primi in forma sillogistica. Secondo questi interpreti, in questo capitolo delle Categorie siamo in presenza di un caso particolare di quello stesso processo che consente la deduzione del sillogismo. Sospendendo momentaneamente il grave problema russellinao a proposito della definizione di classi a partire da proprietà, possiamo indicare con $\mathrm{P}(\mathrm{x})$ una proprietà e definire una classe $\{\mathrm{x}: \mathrm{P}(\mathrm{x})\}$. Posta una proprietà $\mathrm{Q}(\mathrm{x})$ definiamo una classe $\{\mathrm{y}: \mathrm{Q}(\mathrm{y})\}$. Ora, dire che la classe $\{\mathrm{x}: \mathrm{P}(\mathrm{x})\}$ include la classe $\{\mathrm{y}: \mathrm{Q}(\mathrm{y})\}$ significa sostenere che "per ogni $\mathrm{z}$, se $\mathrm{z}$ è membro della classe degli $\{\mathrm{y}: \mathrm{Q}(\mathrm{y})\}$ allora è anche membro della classe $\{\mathrm{x}: \mathrm{P}(\mathrm{x})\}$. In altri termini, se la classe nominata dalla proprietà "animale" include la classe nominata dalla proprietà "uomo" e "questo uomo qui" appartiene alla classe nominata dalla proprietà "uomo", allora vale la transitività della proprietà "animale" su "questo uomo qui".

\section{Peculiarità nella formulazione del principio di transitività}

Se quanto detto fino a qui è vero, è legittimo lamentare che l'applicazione della legge di transitività della relazione predicativa al caso delle Categorie, lascia emergere una incongruenza. Ciò è dovuto al fatto che le relazioni messe in luce da

Aristotele nelle Categorie non valgono solo sul piano linguistico, ma soprattutto su quello ontologico. Nel capitolo 2 Aristotele adotta le espressioni "essere predicato di un sostrato" e "stare in un sostrato" come criteri per ottenere una classificazione degli enti e non una classificazione di itens linguistici. "Essere predicato di un sostrato" indica una relazione intracategoriale fra gli enti; mentre "stare in un sostrato" indica una relazione intercategoriale tra una ousia e un accidente. In generale, nelle Categorie Aristotele non sembra essere interessato 
nei tipi di relazioni logiche che si stabiliscono tra i tipi di predicati e il soggetto a cui sono attribuiti. Aristotele è interessato a distinguere i predicati che sono in grado di identificare in modo rilevante e soddisfacente il soggetto a cui sono attribuiti, ossia i predicati essenziali, e i predicati che non sono in grado di operare questa identificazione, ossia i predicati eterogenei.

Ora, poiché la relazione del 'dirsi di' deve essere transitiva, deve essere univoca. Ma ciò non vale nel nostro caso, poiché dirsi "uomo" di "questo uomo qui" esprime una relazione di inclusione di un individuo in una classe, mentre dirsi "animale" di "uomo" esprime una relazione di appartenenza della specie alla classe rappresentata dal genere ${ }^{7}$. In altri termini, sono presenti due valenze del 'dirsi di'. Dire 'questo uomo qui è uomo' e 'uomo è animale' non si equivalgono dal punto di vista logico. La specie si predica di un individuo in un modo diverso da come il genere si predica della specie.

Per Ackrill (Ackrill 1963, 77), la differenza consiste nella diversa funzione che il termine 'uomo' riveste nei due casi: in un caso funge da soggetto, in un caso funge da predicato. Per Ackrill, siamo, perciò, in presenza di una differente valenza del termine medio nelle due premesse del sillogismo. Per Patzig (Patzig 1968, 108), la diversità è dovuta al fatto che il termine 'è' assume un significato differente: da una parte esprime l'appartenenza; dall'altra la subordinazione di una classe rispetto ad un'altra. Da una parte abbiamo un rapporto tra un elemento e la sua classe di appartenenza; dall'altra abbiamo un rapporto di inclusione tra classi. Quindi non abbiamo a che fare con una relazione, ma propriamente parlando, con due relazioni. E con ciò viene meno la condizione di transitività.

Da parte nostra, crediamo che le cose stiano in un modo leggermente differente. Cerchiamo, pertanto, di utilizzare il principio della transitività in modo tale da evitare di attribuire incongruenze al testo delle Categorie. Schematicamente il principio afferma quanto segue:

$\mathrm{Y}$ è predicato di $\mathrm{X}$ come di un sostrato $\& \mathrm{Z}$ è detto di $\mathrm{Y} \rightarrow \mathrm{Z}$ è predicato di $\mathrm{X}$

Secondo l'interpretazione tradizionale, il principio afferma la transitività della relazione "dirsi-di". Questo comporta che le espressioni "predicato di" e "detto di" rivestano il medesimo significato. E ciò sembra confermato dagli esempi formulati da Aristotele:

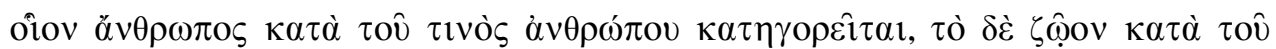

${ }^{7}$ Cf. Ackrill 1963, 76-77. 


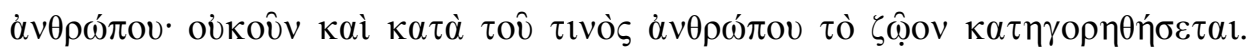

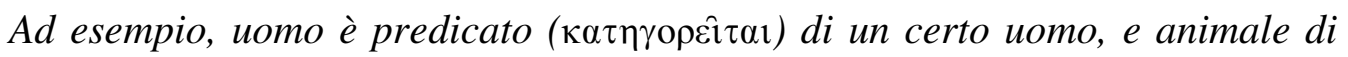

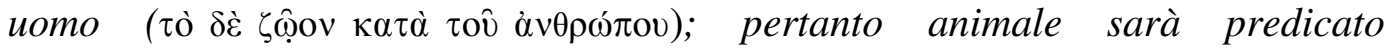

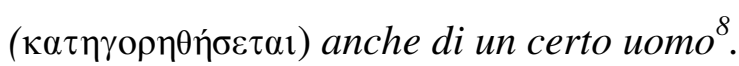

Anzitutto è necessario prestare attenzione alle espressioni utilizzate. Fin dall'esordio delle Categorie ci troviamo di fronte ad una molteplicità di espressioni: legesthai, katêgoreisthai, kath'hupokeimenou legesthai, katêgoreisthai hôs kath'hupokeimenou, e la maggior parte di queste espressioni è utilizzata nei primi cinque capitoli delle Categorie. Per cogliere il senso dell'espressione hôs kath'hupokeimenou, che appare nel capitolo terzo, è importante distinguere fra "predicabili" e "struttura proposizionale soggetto/predicato".

Successivamente, è importante notare che, benché l'esempio di Aristotele affermi esplicitamente che "uomo" e "animale" sono predicati di "questo uomo qui", la relazione tra "animale" e "uomo" rimane implicita.

Illustriamo separatamente i due punti partendo dal primo, ossia la peculiarità delle espressioni utilizzate. Noi crediamo che l'espressione kath'hupokeimenou legesthai non corrisponda qui alla tradizionale struttura "dirsi di un soggetto". Nel capitolo 2 Aristotele adotta le espressioni "essere predicato di un sostrato" e "stare in un sostrato" come criteri per ottenere una classificazione degli enti e non una classificazione di itens linguistici. "Essere predicato di un sostrato" indica una relazione intracategoriale fra gli enti; mentre "stare in un sostrato" indica una relazione intercategoriale tra una ousia e un accidente. In generale nelle Categorie Aristotele non sembra essere interessato nei tipi di relazioni logiche che si stabiliscono tra i tipi di predicati e il soggetto a cui sono attribuiti. Aristotele è interessato a distinguere $\mathrm{i}$ predicati che sono in grado di identificare in modo rilevante e soddisfacente il soggetto a cui sono attribuiti (e questi sono i predicati essenziali), e i predicati che non non soddisfano questa esigenza (predicati eterogenei). Il filosofo sembra concentrato in questa differenza e nella struttura del mondo che essa rappresenta: da un lato egli distingue le sostanze (sostrati primitivi), definiti dai predicati essenziali; dall'altro le proprietà accidentali. Essere affermato (o predicato) di un sostrato è una espressione utilizzata nel capitolo 2 delle Categorie per indicare la predicazione essenziale o sinonima,

\footnotetext{
8 Arist., Cat. 1b12-14.
} 
caratterizzata appunto dal principio della transitività. Y è predicato in modo sinonimo di x e x' se, e solo se, il nome Y e la corrispondente definizione sono predicati di x e x'.

Passiamo al secondo punto, ossia alla relazione fra $\mathrm{i}$ termini utilizzati

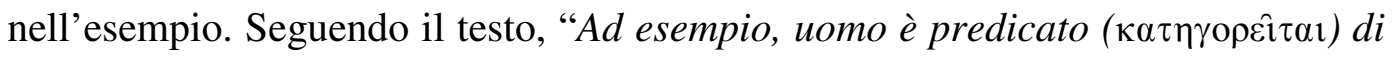

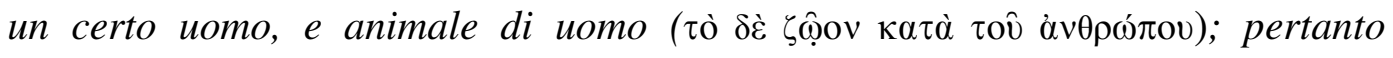

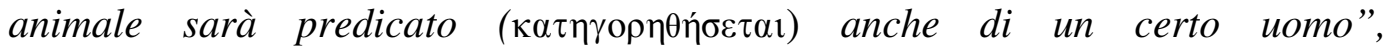
dall'espressione "animale di uomo" risulta "animale è predicato di uomo" e, pertanto, il passo in generale non sembra riconoscere alcuna distinzione fra "predicato di" e "detto di". Se, tuttavia, l'esempio illustra il principio della transitività ("Quando una cosa si predica di un'altra come di un sostrato

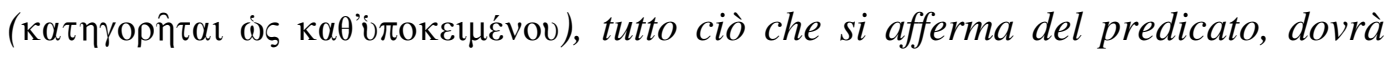
essere affermato anche del sostrato") l'espressione "animale di uomo" genera "animale è detto di uomo", e "predicato di" non necessariamente riveste lo stesso significato di "detto di". Con ciò non vogliamo negare che Aristotele creda che "uomo è detto di questo uomo qui". Il punto è che, il principio enunciato dallo Stagirita potrebbe rivestire anche una funzione diversa all'interno delle Categorie.

Del resto, se l'obiettivo di Aristotele è di limitarsi ad enunciare il principio della transitività della relazione "dirsi di", per quale ragione affermare che "uomo è predicato di questo uomo qui "come di un soggetto"? Inoltre, il filosofo avrebbe realmente ignorato la differenza nella relazione che corre tra un individuo e la specie alla quale un individuo appartiene e la relazione tra la specie e il suo genere.

\section{Principío di inclusione e principio di esclusione}

Tentiamo ora di interpretare il testo senza dover ricorrere all'accusa di trascuratezza nei confronti dello Stagirita. Se applichiamo alle osservazioni sul primo punto il principio della transitività enunciato nel secondo punto, possiamo affermare che è solo per il fatto che Socrate e Corisco sono enti concreti che appartengono alla specie "uomo" (classe che si predica in modo sinonimo a Socrate e Corisco), che tutto ciò che è detto di "uomo" può essere predicato di Socrate e Corisco e questo vale per tutti i predicati che, dalla specie "uomo" salgono fino al genere ultimo al di là del quale non è più possibile procedere, 
ossia, in questo caso la categoria della sostanza ${ }^{9}$. Il principale valore del principio della transitività all'interno delle Categorie è il fatto stesso di permettere di relazionare gli individui concreti alle loro categorie corrispondenti, senza che venga perduta la relazione fra l'individuo, o sostanza prima nelle Categorie, e il genere corrispondente al di là del quale non si può ulteriormente procedere, ossia, la categoria corrispondente alla classe degli individui in questione. E questo vale tanto per la categoria della sostanza quanto per le altre categorie. Ritorneremo su questo punto nel seguito del testo.

Alle linee 1b16-20 Aristotele enuncia quella che potremmo chiamare 'legge dell'esclusione ${ }^{10}$, per contrapporla alla precedente legge che potrebbe essere detta 'legge dell'inclusione'. La legge dell'esclusione afferma che specie diverse e non subordinate tra di loro hanno differenze specificamente diverse, ossia la differenza non è di numero ma di natura:

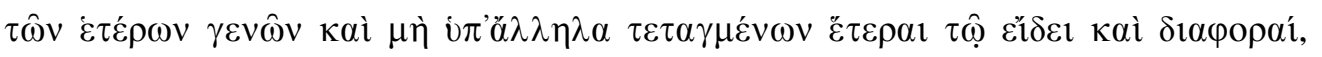

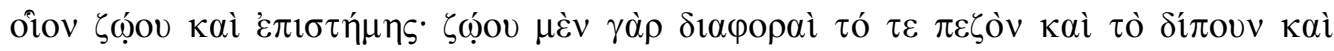

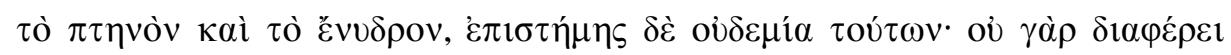

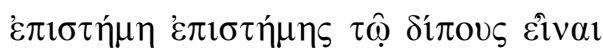

"Dei generi distinti e non subordinati gli uni agli altri le differenza sono specificamente distinte, per esempio, quelle di animale e di scienza. Infatti mentre le differenze di animale sono terrestre, volatile, acquatico e bipede, nessuna di esse è propria della conoscenza; infatti, la conoscenza non differisce da un'altra per il fatto di essere bipede" ${ }^{\prime 1}$.

Alle linee 1b16-20 il filosofo nota che se una stessa differenza convenisse a due specie diverse e non subordinate fra loro, una stessa specie apparterrebbe a due generi diversi e non subordinati.

Paradigmatico a questo proposito è il passo dei Topici VI 6: "Occorre altresì considerare se la differenza nominata si applichi ad un altro genere che non è contenuto nel genere nominato, né lo contiene; in realtà pare che la medesima differenza non debba applicarsi a due generi, nessuno dei quali contiene l'altro. In caso contrario, anche la medesima specie verrà a trovarsi in due generi, nessuno dei quali è nell'altro. In effetti, ciascuna differenza, involge il proprio genere, così come le nozioni di terrestre e di bipede coinvolgono la nozione di

\footnotetext{
${ }^{9}$ Lo stesso procedimento è valido per la deduzione delle altre categorie. Cfr. Wedin 2000, pp. 24-37, in particolare p. 24.

${ }^{10}$ Cfr. Pesce 1966, 32.

11 Arist., Cat. 1b16-20.
} 
animale. Di conseguenza, se di ciò di cui si predica la differenza si predica pure ciascuno dei due generi, evidentemente la specie si troverà in due generi, nessuno dei quali contiene l'altro" ${ }^{12}$.

Ora, è necessario aggiungere, come Aristotele afferma nel seguito, che una stessa differenza può appartenere a due specie diverse e non subordinate tra loro, a patto che entrambe appartengano al medesimo genere: "O per meglio dire, non è impossibile che la medesima differenza si applichi a due generi, nessuno dei quali contiene l'altro; perché la cosa sia impossibile bisogna aggiungere piuttosto: quando tali generi non siano entrambe subordinati ad un medesimo genere. L'animale terrestre e l'animale volatile sono infatti generi contenuti uno nell'altro, eppure la nozione di bipede è differenza di entrambe. Bisognerà dunque aggiungere: quando $i$ generi non siano entrambe subordinati ad un medesimo genere, dal momento che entrambe i generi suddetti sono subordinati alla nozione animale. $\grave{E}$ pure chiaro che la differenza non involge necessariamente il proprio genere, dato che la medesima differenza può applicarsi a due generi non contenuti l'uno nell'altro: risulta così che essa piuttosto coinvolge necessariamente uno solo dei due generi e tutti $i$ generi superiori a questo, come la nozione di bipede coinvolge o la nozione di animale volatile, oppure quella di animale terrestre" ${ }^{\prime 13}$.

Ciò che importa è che $\mathrm{i}$ due generi siano subordinati ad un medesimo genere. Pertanto, è possibile che la medesima differenza si applichi a due generi non contenuti l'uno nell'altro, purché la differenza considerata non sia l'unico tratto definitorio della specie, ossia la specie non venga definita propriamente ed unicamente dalla caratteristica espressa dalla differenza. Se la specie è contraddistinta e definita da più di una nota distintiva, nulla vieta che una differenza sia in comune con un'altra specie ${ }^{14}$.

\section{Incongruenze nell'uso del termine "differenza"}

Apparentemente, Aristotele utilizza nelle Categorie due differenti accezioni del termine "differenza", ossia tratta le differenze sia come attributi, sia come

\footnotetext{
12 Arist., Top. 144b12-20.

13 Arist., Top. 144b20-30.

${ }^{14}$ È interessante constatare che questa regola nei Topici I 15 è utile per distinguere i casi di omonimia. Un termine è omonimo se la nozione da esso espressa è la differenza specifica di due generi diversi e non subordinati l'uno all'altro. 'Acuto' è omonimo della voce e degli angoli, ma la differenza indicata dal termine 'acuto' risulta differente nei due generi distinti.
} 
sostanze. Per molti interpreti, lo statuto che Aristotele attribuisce alle differenze nelle Categorie è piuttosto controverso. Lennox, per esempio, osserva: "the categorical status of diaphora in the Categories is left entirely unclear. Like kinds, differentiae and their accounts may be said of forms and of individuals, but they are not granted even secondary substancehood. Nor are they ever mentioned as belonging to any other category" ${ }^{\prime 15}$. Questo tipo di considerazioni trova conferma nel testo di Aristotele. Nel capitolo 2 delle Categorie, per esempio, nel passo relativo alla quadripartizione degli enti, Aristotele definisce la sostanza prima ed introduce, successivamente, solo genere e specie fra le sostanze seconde. Ora, analoghe a genere e especie sono anche le differenze: se è vero che a 2b30-31 si afferma che genere ("animale") e specie ("uomo") sono "le sole, tra le cose predicate, che manifestano la sostanza prima", mentre le altre determinazioni faranno riferimento alle altre categorie ("bianco", qualità; "seduto", giacere; e così via), che dovremmo dire di "greco" o "egiziano"? Quelle che potremmo chiamare le "sottospecie", ossia le specificazioni ulteriori della classe denominata "specie", non saranno forse analoghe alle sostanze seconde? Esse non sono sostanze, ma si comportano in modo analogo ai generi e alle specie: anche esse, infatti, non sono fra le cose che sono in un soggetto, come è detto esplicitamente a 3a21-25: “questo però non è proprio della sostanza, ma anche la differenza è tra le cose che non sono in un soggetto: infatti terrestre e bipede sono sí detti di un soggetto, ma non sono in un soggetto, infatti non è nell'uomo che è il bipede né il terrestre". Se le differenze mostrano la determinazione propria delle sostanze, perché Aristotele non fa anche di esse delle sostanze seconde? E se le differenze sono sostanze, perché dire "non è proprio della sostanza" ma "anche della differenza" ${ }^{\prime 16}$ ? Oltre a ciò, vi è un'altra caratteristica tipica delle sostanze seconde che, non a caso, appartiene anche alle differenze: la predicazione delle differenze è tipicamente sinonimica: "anche la definizione della differenza è predicata di ciò di cui è detta la differenza: ad esempio, se terrestre è detto di uomo, anche la definizione di terrestre sarà predicata di uomo, infatti l'uomo è terrestre" ${ }^{17}$. Ora, la predicazione è detta sinonimica nel caso in cui A e B appartengano alla stessa categoria. Solo in questo caso, infatti, se A è predicata di B, allora anche la

\footnotetext{
15 Lennox 2001, 165.

16 Arist., Cat. 3 a21.

17 Arist., Cat. 3a28.
} 
definizione di A sarà predicata di B. Le differenze si comportano in modo del tutto analogo a genere e specie.

Alle linee b16-20 Aristotele utilizza una accezione del termine "differenza", secondo la quale "differenza" di un genere è precisamente ciò che divide un genere in generi ad esso subordinati o in specie subordinate al genere. In questo senso, la distinzione tra genere e differenza è relativa: "bipede" è differenza in relazione ad "animale", ma in relazione ad una specificazione ulteriore, può essere assunta come genere, ossia una sostanza seconda, a sua volta suscettibile di suddivisione. Ora, cosa permette ad Aristotele di affermare che, in relazione ad "uomo", "animale" è il genere e "bipede" è la differenza? Niente altro che un criterio di estensione classificatoria: il genere è la classe più ampia, che permette ulteriori specificazioni, mentre la differenza è precisamente ciò che introduce nel genere una nota più specifica $\mathrm{e}$, pertanto, possiede una minore estensione.

In una accezione distinta del termine "differenza", essa è la caratteristica specifica, ossia il tratto che caratterizza specificamente un genere.

Noi crediamo di poter mitigare un po' le preoccupazioni degli interpreti in relazione a questa apparente negligenza nell'uso del termine "differenza" analizzando più attentamente la funzione delle differenze nelle Categorie. A nostro avviso, la presenza delle differenze nel capitolo 3 delle Categorie è giustificata dal fatto che esse permettono di costruire la struttura intra-categoriale di cui il filosofo necessita per strutturare la tavola delle categorie, attraverso l'uso del principio della transitività. Le differenze permettono la progressiva sostituzione del genere superiore senza perdere il contatto con gli enti concreti che appartengono alla classe di cui la specie è sinonimicamente predicata, fino a che si raggiunge il termine finale: la categoria propria cui l'ente appartiene.

$$
\begin{aligned}
& d G(G=\operatorname{sostanz} a) \\
& d, " ' G \text {,', } \\
& d^{\prime \prime} G^{\prime \prime} \\
& d^{\prime} G^{, 18}
\end{aligned}
$$

Socrate è "иomo"

Il principio della transitività relaziona gli individui concreti, attraverso la predicazione sinonimica, fino alla categoria cui essi appartengono. Ciò è possibile costruendo un albero genealogico ascendente di differenze+generi. Se si

\footnotetext{
${ }^{18} d$ sta per "differenza specifica", $G$ sta per "genere prossimo".
} 
sostituisce la specie "uomo" con la definizione "differenza+genere", $d$ ' $G$ ', e si sostituisce, a sua volta, questa definizione con la definizione $d G$ di quest'ultima specie, si arriva al genere sommo che si trova al di sopra di una collezione completa di differenze ${ }^{19}$. La scelta di sostituire progressivamente i generi subordinati con le definizioni "differenza + genere" è rafforzata da altri testi, in cui lo Stagirita difende la correttezza delle definizioni governate dalla coppia "genere+differenza". In Metafisica VII 12 il filosofo dichiara che la definizione dovrà contenere nient'altro che "il genere primo < primo in una divisione>" e la differenza $^{20}$. Negli Analitici Posteriori Aristotele offre indicazioni precise per condurre correttamente le divisioni in modo tale da individuare correttamente gli stadi gerarchici nel cammino che conduce dagli individui concreti ai generi e viceversa $^{21}$. Nei Topici il filosofo fa notare che il genere e le differenze di una specie sono maggiormente intelligibili e prime in relazione alla specie $^{22}$. La nozione di priorità cui Aristotele si richiama in questo testo è certamente legata alla posizione in cui un termine si trova all'interno della scala gerarchica, ma possiede anche un valore ontologico: se il genere e la differenza venissero distrutti, la specie, a sua volta, scomparirebbe. La struttura gerarchica permessa dal principio di transitività riflette certe relazioni ontologiche.

Il procedimento della progressiva sostituzione $d G$ ci fornisce una mappa che parte dagli individui e arriva alle categorie. Seguendo l'esempio dell'albero genealogico ascendente, si parte da Socrate e si arriva alla conclusione che questo individuo è $d$ ', $d$ ', $d$ ' ', '....... $d^{n}$ sostanza. Nel libro II degli Analitici Posteriori, al capitolo 13, Aristotele descrive come avviene il processo della successiva differenziazione ${ }^{23}$. Benché il passo rientri in un contesto differente e rivesta una differente funzione rispetto all'obiettivo in vista nelle Categorie, ci pare utile citarne il contenuto, al fine di mostrare come la preoccupazione di Aristotele sia anche in quest'occasione quella di individuare e isolare il genere all'apice senza perdere il contatto con gli elementi costituenti, a partire dalle sostanze prime delle Categorie, ossia gli enti naturali. Anzitutto, stabilisce Aristotele, le differenze devono essere predicati essenziali. L'espressione utilizzata da Aristotele è

\footnotetext{
${ }^{19}$ Cfr. Wedin 2000, 24-34.

${ }^{20}$ Arist., Metaph. VII 12, 1037b27-30.

${ }^{21}$ Arist., $A$ Po II 13, 96b15-97a6.

22 Arist., Top. $141 \mathrm{~b} 27-30$.

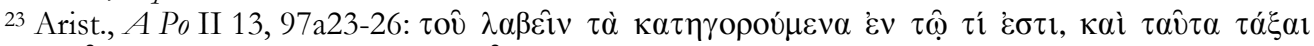

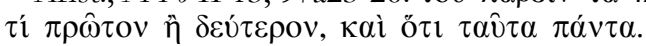




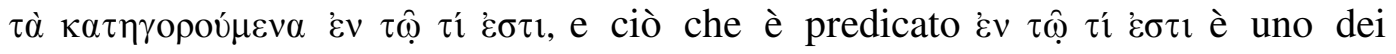
generi del predicato essenziale ${ }^{24}$. Nel capitolo quarto degli Analitici Secondi Aristotele delimita i tipi di attributo, o predicato, che possiamo definire "predicati essenziali" ${ }^{25}$. Si tratta degli attributi che mantengono con il soggetto una relazione necessaria, o "attributi per sé". Gli "attributi per sé" si distinguono in due tipi: il primo é tale che, dato il soggetto, necessariamente segue il predicato; il secondo é tale che, dato il soggetto, segue come attributo una disgiunzione (o tale predicato o il suo opposto). Inoltre, il primo tipo appartiene alla struttura interna del soggetto e manifesta un contorno più nitido, dal momento che si tratta dell'attributo che è incluso nell'enunciato che definisce ciò che è qualcosa, ossia l'attributo che appare in una predicazione essenziale ${ }^{26}$. Il secondo, invece, non costituisce la struttura interna del soggetto. Seguendo la dicitura delle Categorie, il primo tipo di attributo comprende le "sostanze seconde" e le differenze.

In secondo luogo, la divisione deve procedere in modo successivo, dal momento che questo è l'unico modo per ordinare i predicati successivamente in una linea di divisione.

Infine, la divisione deve essere completa, ossia deve procedere fino al punto in cui una ulteriore divisione produrrebbe differenze non naturali ma arbitrarie.

Solo delimitando in questo modo una unica linea corretta di divisioni risulta possibile stabilire la natura del genere in modo non arbitrario ${ }^{27}$.

\section{La predicazione delle differenze}

Nell'ultima parte del capitolo 3 Aristotele avanza alcune considerazioni che hanno lasciato perplessi gli interpreti. Alle linee b21-22 il filosofo afferma che nel caso in cui i generi siano diversi ma subordinati gli uni agli altri, è possibile, ma non necessario, che le differenze del genere siano le differenze del genere ad esso subordinato:

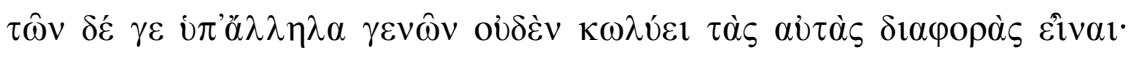

"Invece dei generi subordinati gli uni agli altri niente impedisce che le differenze siano le stesse" 28 .

\footnotetext{
${ }^{24}$ Arist., $A$ Po I 4, 73a34-b5.

${ }^{25}$ Cfr. Arist., $A$ Po I 4, 73a24-25.

${ }^{26}$ Cfr. Arist., Top. 102a32-35 e I 9. È il tipo di attributo che significa che cosa è il soggetto (Top. 103b27-35), e significa una sola cosa (Metaph. IV 4, 1006b18).

${ }^{27}$ Cfr. Arist., Top. 141a34-b2.

28 Arist., Cat. 1b22-24.
} 
Ciò è possibile ma non è necessario, dal momento che alcune proprietà del genere possono essere contenute in una particolare proprietà che caratterizza il genere subordinato. Per esempio, nell'affermazione 'l'uomo è bipede', all'interno della differenza 'bipede' è contenuto 'terrestre'. Possiamo affermare 'l'uomo è terrestre', ma nell'affermazione 'l'uomo è bipede', la differenza 'terrestre' è già contenuta. Come fa notare Boezio, il genere 'animale' e il genere 'uccello' sono generi diversi e subordinati l'uno all'altro: 'uccello' è subordinato ad 'animale'. Ora, a seconda delle differenze che si assumono per animale, le stesse possono convenire o meno ad 'uccello'.

In generale, il fondamento dell'affermazione di Aristotele alle linee 1b21-22, ossia il fatto che sia possibile che una medesima differenza convenga a due specie tra loro subordinate, risiede nel principio della transitività e in particolare alle linee 1b10-13: ciò che è detto del predicato è detto anche del soggetto ${ }^{29}$. Poiché la differenza si dice della specie che essa determina e la specie si dice della sottospecie, allora anche la differenza si dice della sottospecie.

In verità, secondo la definizione di "differenza" come il tratto che contraddistingue una specie nel continuum de genere, la presente affermazione di Aristotele non è del tutto giustificata. Noi crediamo che in questo passo sia più corretto parlare di "proprio", piuttosto che di "differenza" in generale. Il "proprio", infatti, non si annovera tra le parti della definizione, e le proprietà che convengono alla sottospecie in virtù del fatto che convengono alla relativa specie, non sono fra le parti che definiscono tale sottospecie. Il "proprio" è un tipo di predicato all'interno del quale sono contenute anche le differenze specifiche. Il "proprio" è distinto dalla nozione di differenza che per comodità, chiamiamo “differenza non propria" in questo senso: la "differenza non propria" è un tipo di predicato che costituisce l'essenza del soggetto; inoltre, il soggetto implica il predicato, mentre il predicato non implica il soggetto; il "proprio", a sua volta, non costituisce l'essenza del soggetto ed è un tipo di differenza di cui si contropredica il soggetto a cui viene attribuito.

All'interno del "proprio" sono contenute le differenze specifiche, così come esse sono concepite in Metafisica VII 12, ossia come termine in cui si completa una serie di divisioni che, ad ogni tappa, assume un attributo che essenzialmente

\footnotetext{
${ }^{29}$ La differenza è fra le cose 'dette di' e che non 'sono in', Cat. 5, 3a22-28.
} 
contiene tutte le differenze anteriori. Così concepita, la differenza porta ad un risultato paradossale. Infatti, la differenza ultima, rappresenterebbe la definizione stessa dell'ente, indipendentemente dalla presenza del genere. Ciononostante, lo Stagirita, non sembra essere turbato da questa conseguenza e, al contrario, la annuncia con un certo orgoglio ${ }^{30}$.

Aristotele giustifica quanto detto alle linee b20-21 con na conclusione quanto meno curiosa:

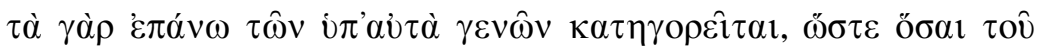

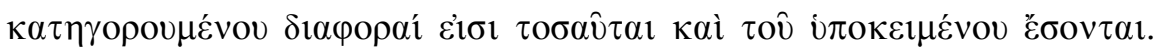

"Infatti quelli superiori si predicano dei generi ad essi subordinati, cosicché quante sono le differenze del predicato altrettante saranno anche le differenze del sostrato". 31

Il senso del testo non può essere che tutte le differenze del genere si predicano di tutte le specie. Nel caso in cui si assuma la "differenza non propria", se essa ha la funzione di dividere il genere, ad ogni specie converrà la determinazione di carattere comune del genere, ma non anche tutte le determinazioni che contraddistinguono il genere. A queste due linee sono state fornite esegesi differenti. Forniamo le principali.

Ackrill si muove lungo una linea esegetica secondo la quale le differenze cui Aristotele si riferisce in questo passo non sono tutte le differenze del genere, ma quelle che originano una determinata specie. Questi attributi, individualmente selezionati, appartengono anche a membri di altre specie subordinate allo stesso genere, ma una accurata lista delle differenze di una specie apparterrà, collettivamente considerata, solo ai membri di quella specie ${ }^{32}$. Per esempio, se si tratta della specie "uccello", si farà riferimento a tutte le differenze di animale che concerno la specie uccello: volatile, oviparo, bipede, piumato e così via. Utilizzando la terminologia scolastica Ackrill distingue in "differentiae divisivae" e "differentiae constitutivae". Secondo Ackrill, le differenze costitutive di una specie si annoverano anche tra le differenze dei corrispondenti generi sovraordinati. Lo studioso fondamenta la propria ipotesi sul passo 1b16-20, nel quale Aristotele attribuisce al genere animale la proprietà bipede come differenza,

\footnotetext{
30 Arist., Metaph. 1038a19-20; 1038a25-26; 1042b9. Questo aspetto curioso e controverso della dottrina aristotelica è analizzato e sviluppato da Angioni 2000, 521-48.

31 Arist., Cat. b22-24.

32 Cfr. Arist., A Po II 13, 96a24-34.
} 
benché tale proprietà costituisca una differenza di una specie del genere animale. Questa interpretazione è stata criticata dallo Oehler, secondo il quale le differenze che non abbracciano un genere, ma solo lo dividono in specie, non possono essere dette di questo genere. Perciò "bipede" non può essere predicato di animale, perché non ad ogni animale conviene di essere bipede. La differenza deve avere la capacità di abbracciare anche il genere, deve essere la differenza costitutiva del genere prima di tutto.

Una seconda interpretazione, che era quella sostenuta da Boezio, parte dal presupposto che differenze di cui parla Aristotele sono appunto "differentiae constitutivae" in rapporto al genere predicato e tali differenze si applicherebbero anche alla specie. Secondo Boezio, queste differenze non sono quelle che suddividono il genere ("generis divisivae"), ma quelle "specificae et completivae", ossia che appartengono alla stessa determinazione. Se il termine cui vengono predicate le differenze è "animale", si tratta delle differenze "sensibile", "animato", e così via. Ackrill accetta, in un secondo momento questa lettura: poiché la differenze del genere predicato devono essere differenze della specie di cui esso è predicato, questo può verificarsi solo se le differenze definiscono sia il genere, sia la specie. Pertanto, l'argomento delle ultime linee è valido solo se si considera che "differenza" indica la differenza definitoria. È vero, tuttavia, che gli esempi che il filosofo offre nelle linee anteriori del testo sono casi di differenze divisorie. Perciò, dovremmo concludere che Aristotele transita inavvertitamente da un significato all'altro del termine "differenza".

Pesce ritiene, in generale, che queste interpretazioni siano inappropriate, dal momento che il testo non menziona queste distinzioni.

Un terzo modo di interpretare il testo, sarebbe quello di intendere non solo le differenze costitutive del genere, ma tutte le differenze, e fondamentalmente le differenze divisivae, ciascuna delle quali si dice della specie che in corrispondenza ad essa si determina, perciò le differenze del genere sono le differenze di tutte le specie in senso distributivo. Questa lettura mira a non perdere l'accentuazione della corrispondeza fra le differenze del genere e quelle della specie che Aristotele esprime con i correlativi "hosai" ... "tosautai". Tuttavia, ci pare che questa interpretazione si presti alle stesse critiche mosse alla prima esegesi. 
Oehler, da parte sua, chiarisce anzitutto la nozione di "differenza", distinguendo fra "proprietà possibili" e "proprietà caratterizzanti e definienti". Questa distinzione varia con il variare dei termini presi in considerazione, ossia, "bipede", in relazione alla specie "uomo" è differenza caratterizzante e definiente", in relazione al genere "animale" è proprietà possibile. Le proprietà che caratterizzano una specie costituiscono i "propri” di tale specie; le proprietà possibili comprendono sia i "propri”, sia proprietà accidentali.

Un'altra soluzione possibile potrebbe essere quella di credere che l'argomento, al contrario di ciò che il contesto suggerisce, non si fondi sul principio della transitività e che le espressioni "del genere predicato" e "del soggetto" nel testo in questione sono dislocate, ossia, l'una sta al posto dell'altra. In questo caso non si avrebbe ambiguità nell'uso del termine "differenza", ossia Aristotele avrebbe in mente solo il senso "divisorio" del termine differenza e ciò risulterebbe in armonia con gli esempi forniti nelle linee anteriori del testo.

In conseguenza all'analisi delle interpretazioni fornite dagli studiosi, possiamo tratte le seguenti conclusioni.

Anzitutto, alle linee 1b21-22, Aristotele afferma che quando un genere è subordinato ad un altro è possibile che le differenze dell'uno siano differenze dell'altro. Questa frase, così come è stata formulata e nel contesto in cui è inserita, suggerisce la seguente lettura: se A è un genere subordinato di B, B si predica di A. Ora, seguendo la transitività della relazione predicativa, tutto ciò che si predica di B si predica ugualmente di A; pertanto le differenze di B saranno anche differenze di A. Il suddetto argomento suppone che le differenze di un genere G si predicano del genere stesso. Questo, tuttavia, non collima con gli esempi che Aristotele utilizza nelle linee che precedono immediatamente, ossia le linee 1b1620. Infatti, terrestre, volatile, acquatico e bipede sono differenze del genere "animale" e sono predicate di generi ad esso subordinati, ma non si predicano di animale. Perciò, il termine "differenza" alle linee b21-22 non può indicare la differenza che divide il genere in generi subordinati, bensì il "proprio". Ora, sono le differenze del genere subordinato che sono anche differenze del genere superiore, e non l'inverso. Per esempio, "terrestre" è una differenza del genere "animale" e definisce un genere subordinato; questo genere subordinato, a sua volta, si divide in genere inferiori, rispettivamente attraverso le differenze "bipede", "quadrupede", ecc. Ora, bipede e quadrupede sono differenze del genere 
subordinato "animale terrestre", ma allo stesso tempo sono differenze del genere "animale",33.

Assumiamo la differenza "bipede" (D). "Bipede" si predica del soggetto "uomo" (S), non è un attributo contenuto nella definizione del soggetto, ma decorre necessariamente dal soggetto a cui è attribuito, si tratta quindi di un "proprio". Lo stesso attributo di predica del genere, "animale terrestre" (G). La differenza $D$ è in relazione a $G$ un attributo per sé di secondo tipo congiunto (gli animali terrestri sono bipedi o quadrupedi). Ora, dato che questo tipo di predicato, quando assume congiuntamente le opzioni che esauriscono le sue possibili specificazioni si rende contropredicabile con il soggetto, non differisce dall' attributo "proprio" 34 . In questo caso, la differenza del genere si predica tanto del soggetto "uomo", quanto del genere "animale terrestre" 35 . È difficile decidere se la sola caratteristica della contropredicabilità sia sufficiente a rendere un "attributo per sé di secondo tipo" un "proprio". È già stato versato molto inchiostro in merito alla questione della relazione tra questo tipo di attributo e ciò che nei Topici Aristotele denomina "proprio" (idion) ${ }^{36}$. Intorno ai possibili legami tra questo tipo di attributo e le differenze in relazione al genere è interessante lo studio di Ferejohn, The origins of Aristotelian science ${ }^{37}$. Si potrebbe forse affermare che questo tipo di "attributo per sé" costituisce una classe più ampia di cui il "proprio" sarebbe una specificazione. La caratteristica comune ad entrambe i tipi di predicato è il fatto che ciascuno è attribuito esclusivamente al suo proprio soggetto. Tuttavia, se un "attributo per sé" fosse considerato in unione con il suo opposto complementare, la coppia di contraddittori formatasi potrebbe essere considerata come un "predicato proprio". Per esempio, nella frase "la linea è retta o curva", se il predicato F fosse sostituito dalla frase "retta o curva", avremmo un predicato proprio della linea. Se invece F fosse sostituita da uno solo degli opposti, avremmo un attributo"per sé" della linea.

\footnotetext{
33 Angioni 2009, pp. 38-40; 170-171.

34 Angioni 2009, p. 39.

${ }^{35}$ L' "attributo per sé di secondo tipo" é presentato da Aristotele nei termini seguenti: questi predicati sono tali per cui, nei loro rispettivi enunciati definitori, é necessario menzionare i soggetti ai quali appartengono. Cfr. 73a37-38, 84a13-14, 186b20-21, 1030b23-25.

36 Arist., Top. 102a18-19. Vd. Angioni 2009, pp. 38-40 ; 170-171 ; Barnes 1993, pp. 113-114, Tiles 1983, Code 1986, p. 350 ; Hadgopoulos 1976.

${ }^{37}$ Ferejohn 1991, pp. 99-108.
} 
Come ha notato Angioni, di fatto, lo Stagirita non sembra aver preso coscienza di queste possibilità. Quando definisce la nozione di attributo proprio, in Topici I 5 , il filosofo si fonda solo sul criterio della contropredicabilità ${ }^{38}$.

Infine, la nostra suggestione è che se per ogni differenza che decorre necessariamente dal soggetto di cui si predica, e che non sia inclusa nella sua definizione, fosse possibile trovare un livello di relazione predicativa in cui essa figuri come attributo di secondo tipo, avremmo il caso in cui la stessa differenza si predicherebbe tanto del genere quanto della specie ad esso subordinata.

\section{Bibliografia}

J. L. Ackrill, Aristotle's Categories and De Interpretatione, Oxford 1963.

L. Angioni, Introdução à Teoria da Predicação de Aristóteles, Campinas 2009.

H. G. Apostle, Aristotle's Categories and Propositions (De Interpretatione), Oxford 1975.

A.T. Bäck, Aristotle's theory of predication, Leiden 2000.

R. Bodéüs, Aristote - Catégories, Paris 2002.

J. Dürlinger J, Predication and Inherence in Aristotle's Categories, «Phronesis» 16 (1970), 179-203.

E. D. Harter, Aristotle on primary ousia, «Archiv für Geschichte der Philosophie»

J. Hintikka, Aristotle on Modality and Determinism, «Acta Philosophica Fennica» 29 (1977), 7-124.

J. G. Lennox, Aristotle's Philosophy of Biology, Cambridge 2001

F.A. Lewis, Substance and Predication in Aristotle, Cambridge 1991.

A. C. Lloyd, Form and Universal in Aristotle, Liverpool 1981.

J. M. E. Moravcsik, Aristotle's theory of categories, in J. M. E. Moravcsik (ed.), Aristotle: a collection of critical essays, New York 1967a, pp. 125-145.

J. M. E. Moravcsik, Aristotle on Predication, «Philosophical Review» 76 (1967b), 80-97.

K. Öhler, Aristoteles, Kategorien, III ed., Berlin 1997.

G. E. L. Owen, Logic and metaphysics in some earlier works of Aristotle, in Martha Nussbaum (ed.), Logic, science and dialectic, Londres 1986a, pp. 180-199.

G. Patzig, Die aristotelische Syllogistik, Göttingen 1968 (II ed.).

D. Pesce, Aristotele, Categorie, Padova 1966.

Porphyre, Isagoge, texte grec et latin, traduction par A. De Libera, Paris 1998.

W. D. Ross, Aristotle, London 1956.

M. V. Wedin, Aristotle's theory of substance, Oxford 2000.

M. Zanatta, Aristotele, Categorie, introd., trad., e commento, Milano 1989.

${ }^{38}$ Ad esso aggiunge appena la caratteristica puramente negativa che non si predica il to ti en einai del soggetto a cui il proprio viene attribuito. 